\title{
Marcel Proust en BD : circonscrire l'adaptation de Stéphane Heuet
}

\author{
François-Emmanuël Boucher \\ Collège militaire royal du Canada
}

Le rapport entre l'œuvre littéraire et son adaptation picturale a toujours été des plus contradictoires tant il semble que certaines œuvres se prêtent naturellement à ce type de pratique alors que d'autres semblent la proscrire pour des raisons qui relèvent à la fois d'une conception spécifique de la littérature et de critères proprement esthétiques. La question centrale serait de savoir pourquoi transposer en image ce qui est d'abord à l'écrit et ensuite se rendre compte que les réponses à cette question sont multiples, qu'elles varient dans le temps et en fonction des médiums employés. 


\section{L'Illustration dans le temps : des jalons}

Par exemple, malgré leur contenu souvent abstrait, rébarbatif et hautement spirituel, l'Ancien et le Nouveau Testaments ont été, à partir du VIe siècle, illustrés en Occident à l'aide de vitraux et de tapisseries. On possède encore aujourd'hui des fragments de mosaïques de verre qui illustrent des scènes des Évangiles qui ornaient l'intérieur de l'abbaye puis de la cathédrale SaintBénigne de Dijon de même que la première cathédrale de Beauvais construite au tournant du Xe siècle. La Bible de Vivien, dite la première Bible de Charles le Chauve, confectionnée à Tours vers 845 , déborde quant à elle d'illustrations que l'on peut toujours voir à la Bibliothèque nationale de France. Ces enluminures et ces dessins d'une grande précision accompagnent la traduction latine de saint Jérôme de manière à mettre sous les yeux des croyants, si ce n'est de l'Empereur luimême, sans doute un parfait illettré, des images édifiantes représentant les scènes du péché originel, de la sortie d'Égypte, de l'annonce faite à Marie, de la crucifixion, etc. Vitraux, dessins, peintures, enluminures, tapisseries, sculptures et portiques participent à ce que l'on nomme traditionnellement l'Évangile du pauvre, c'est-à-dire la fabrication de représentations autant rudimentaires soient-elles de la vie des prophètes, de Jésus et des saints, qui ont pour caractéristique première d'être simples et, surtout, déchiffrables par la vue. Ces représentations qui se veulent accessibles ont une fonction didactique évidente : elles éduquent, elles racontent, elles font voir à celui qui autrement ne pourrait pas accéder au texte biblique. En fait, ces représentations fournissent, sans doute à cette époque éloignée, les seules connaissances que le commun des mortels possédait sur la religion chrétienne, connaissances 
essentiellement minimales et très superficielles. La Bible de Lubeck, fabriquée entre 1533 et 1534, c'est-à-dire la première édition de la Bible de Luther en bas-allemand, nommée aussi Bible de Bugenhagen en honneur de son imprimeur, poursuit en quelque sorte cette tradition qui consiste à illustrer les passages essentiels du récit de la vie et de la mort du Christ. Dans cette première édition, on dénombre 79 illustrations de bois gravé de même qu'un frontispice fait d'une peinture de Lucas Cranach l'Ancien. L'austérité protestante n'implique en rien de faire l'économie des représentations: la transmission $\mathrm{du}$ message biblique est quasiment indissociable de son illustration

Dès le début de la Renaissance, ce ne sont plus seulement les plus illustres histoires du Nouveau et de l'Ancien Testaments qui deviennent des thèmes obligés pour le Quattrocento, mais, grâce aux améliorations techniques liées à l'imprimerie, les œuvres dites illustrées deviennent de plus en plus courantes et, surtout, elles ne visent plus strictement l'édification et la piété chez le peuple analphabète. En 1524, Pietro L'Arétin publie I Modi (Les Manières), un livre grivois contenant 16 estampes illustrant diverses positions sexuelles, ce qui en fait le premier livre érotique de l'histoire de l'imprimerie. Au XVII ${ }^{e}$ siècle, les livres illustrés pour enfants font leur apparition. Il y a certes Les Contes de ma mère l'oye (1697) de Perrault illustrés par Nicolas Clouzier, mais aussi le premier recueil des Fables de Lafontaine qui, en 1668, est illustré par le plus grand dessinateur français de l'époque, François Chauveau (1613-1676), qui ne fait pas seulement la transposition en image du Corbeau et du renard, mais illustre tous les grands livres du siècle de Louis XIV : c'est lui qui illustre Artamène ou le Grand Cyrus, qui dessine la Carte de Tendre dans Clélie de Madame de Scudéry, de même que plusieurs œuvres de Scarron, de Molière et de Racine. 
Cette généralisation de la littérature illustrée se poursuit jusqu'au XIX ${ }^{\mathrm{e}}$ siècle, qui est paradoxalement à la fois l'âge d'or de ce type de production (pensons ici seulement à Gustave Doré, qui illustre plus d'une centaine de volumes, dont ceux de Cervantès, de Rabelais, de Dante, de Balzac, de Victor Hugo, de Théophile Gauthier) et l'époque où se manifestent ses premiers détracteurs au nom d'une sacralisation de l'œuvre littéraire et d'une nette démarcation entre les champs artistiques, séparation qui présuppose une forte volonté de hiérarchiser les arts et d'exclure une certaine production littéraire du champ de la démocratisation et de la marchandisation de l'œuvre littéraire, dont l'accessibilité à un vaste public serait le premier critère, et son illustration son vecteur de réalisation le plus commun. La résistance qu'oppose Gustave Flaubert, par exemple, à l'éditeur Lévy qui lui demande, en juin 1862, la permission d'illustrer la première édition de Salammbô, serait représentative de ce nouvel état d'esprit rébarbatif. Son refus outré, radical, indéfectible, vis-à-vis de la volonté de l'éditeur d'illustrer son œuvre carthaginoise, indique une nouvelle sensibilité à l'égard de ce type de pratique, qui apparaît maintenant injurieuse pour certains créateurs. En raison d'une conception de la littérature qui, sans doute, aurait été inintelligible autant à Lafontaine, à Perrault qu'à Voltaire, qui ne connaissaient pas, il faut le dire, la marchandisation hyperbolique de l'art, Flaubert pose comme axiome indiscutable que la grandeur d'une œuvre littéraire est de se suffire à elle-même dans ce territoire abstrait de la pureté esthétique qui relève strictement de l'intellect et non de cette réalité dégradée que présentent les sens mis au service de la raison pécuniaire. D'une logique bienfaisante qui mise sur l'accessibilité dont ce que l'on nomme l'Évangile des pauvres 
serait le paradigme, le $\mathrm{XIX}^{\mathrm{e}}$ siècle fait de l'illustration une pratique au service de la logique bourgeoise et capitaliste. À celui que rebute une telle conception de l'œuvre d'art, cette dernière apparaît intransposable, tant la sphère dont elle relève s'apparente de plus en plus à l'ordre du sacré, c'est-à-dire à une dimension de l'expérience humaine qui ne saurait se prêter à la pratique marchande. Bien qu'il ne parle que de Salammbô, Flaubert fournit par ses explications les principaux topoï à ceux qui, encore récemment, ont été outrés par la transposition en BD de l'œuvre de Marcel Proust en raison du caractère supposément intraduisible du fait littéraire de haute volée. Même si Stéphane Heuet justifie son travail sur Proust à partir d'une logique différente que j'explique plus loin, les arguments de Flaubert tiennent toujours pour les puristes et les tenants de la sacralité de la création littéraire :

Jamais, moi, vivant, [lit-on dans une lettre adressée à Ernest Duplan, en date du 12 juin 1862,] on ne m'illustrera, parce que la plus belle description littéraire est dévorée par le plus piètre dessin. Du moment qu'un type est fixé par le crayon, il perd ce caractère de généralité, cette concordance avec mille objets connus qui font dire au lecteur : "J'ai vu cela» ou «Cela doit être ». Une femme dessinée ressemble à une femme, voilà tout. L'idée est dès lors fermée, complète et toutes les phrases sont inutiles tandis qu'une femme écrite fait rêver à mille femmes. Donc, ceci étant une question esthétique, je refuse formellement toute espèce d'illustration. (Flaubert, p. 221-222)

L'artiste de talent crée une œuvre telle qu'elle ne peut être transposée dans l'ordre du visible sans qu'elle ne se dégrade par la même occasion. Loin de favoriser la lecture ou de rendre un texte plus accessible au commun des mortels, l'illustration littéraire détruit la raison d'être du romancier en la soumettant à la logique des marchands du Temple, qui est aussi celle de Monsieur Homais. L'illustration, selon les dires de Flaubert, 
diminue ce qui est le propre de la littérature, car elle essaie de rendre visible ce qui ne relève que de l'ordre du langage, des mots, d'une conception de la phrase dont rien dans le domaine des arts picturaux ne pourrait être l'équivalent. Le 24 juin 1862, Flaubert écrit encore à Duplan :

La persistance que Lévy met à demander des illustrations me fout dans une fureur impossible à décrire. Ah! Qu'on me le montre le coco qui me fera le portrait d'Hannibal. - Et le dessin d'un fauteuil carthaginois! Il me rendra grand service. Ce n'est guère la peine d'employer tant d'art à laisser tout dans le vague pour qu'un pignouf vienne abolir mon rêve par sa précision inerte. (p. 226)

Cette hostilité flaubertienne, dont on trouve des similarités dans les commentaires condescendants de Charles Baudelaire au sujet de la photographie, pourrait sans aucune difficulté s'appliquer directement à l'entreprise de Stéphane Heuet, qui pousserait un cran plus loin la logique de démocratisation et de dégradation de l'œuvre d'art par sa mise en bande dessinée d'À la recherche du temps perdu, œuvre qui, en raison de caractéristiques formelles, ne se prêterait guère à une telle transposition. Si Salammbô, dixit Flaubert, doit, pour exister, demeurer dans le flou éthéré d'une syntaxe inamovible, si le propre d'une grande œuvre littéraire est d'être imaginée et jamais vue, quoi penser maintenant d'une adaptation en bande dessinée d'une œuvre de sept tomes, de plus de trois mille pages, dans laquelle la réminiscence, la fluctuation du temps et les stratifications narratives bigarrées sont le produit d'une syntaxe complexe, de continuels discours intérieurs et d'interminables commentaires sur les plus subtiles émotions, dont la nature et la forme transparaissent par un constant usage de métaphores utiles autant pour décrire une gamme étrange d'impressions alambiquées que pour camoufler la 
décadence et la joie de cette civilisation aristocratique. Si Flaubert refusait de rabaisser Salammbô aux procédés de la logique marchande, qu'aurait été la réaction de Proust, qui publia le premier tome de la Recherche à compte d'auteur? Cette question hypothétique fait cependant abstraction d'une transformation significative que connaît l'époque actuelle au sujet des grands textes littéraires d'autrefois. Comme l'explique Heuet dans une entrevue, loin d'être pécuniaire, sa visée est avant tout pédagogique. Il s'agit presque d'un retour à la logique de l'Évangile du pauvre, quoique cette fois-ci, il n'est plus question de rendre accessible le message biblique à une population d'illettrés, mais de transmettre un patrimoine culturel à une jeunesse insouciante et qui, le plus souvent, n'a même plus le temps de lire (Baetens, 2004, p. 257). Contrairement aux pratiques du XIXe siècle, Stéphane Heuet ne vise pas à faire de Proust un best-seller - qui pourrait le faire? -, mais de le sortir de l'oubli et de transmettre un patrimoine à une jeunesse considérée comme inculte au regard de la tradition. À une époque où Proust est devenu plus que jamais illisible pour le commun des mortels, le projet d'en illustrer des parties ne serait plus lié à une logique marchande, mais à celle, inédite, de la transmission d'une culture et d'une certaine connaissance littéraire du passé. Dans un premier temps, j'essaierai de situer l'entreprise de Stéphane Heuet à l'intérieur de la tradition du roman illustré et de la bande dessinée littéraire propres au XXe siècle, pour ensuite m'attarder aux caractéristiques de cette transposition de Proust en bande dessinée, qui a elle-même connu des modifications en cours de route. D'Un amour de Swann à À l'ombre des jeunes filles en fleurs, soit les deux premiers tomes du roman de Proust qui ont été, jusqu'à maintenant, adaptés en cinq BD par Stéphane 
Heuet, ce dernier a renouvelé son art de bédéiste pour des raisons liées à la nature du texte proustien et aux contraintes que commande la bande dessinée, dont la forme finit peu à peu par imposer une autre lecture de La Recherche, de ses personnages et de leurs actions.

\section{Une typologie}

Dans Le Monde des livres du 27 mars 2008, Yves-Marie Labé énonce le constat suivant: "Jacques Tardi a fait des émules : de plus en plus, des éditeurs et des auteurs BD puisent aux fonds littéraires, classique ou contemporain. En 2007, sur les 3312 nouvelles BD publiées en France, 96 sont adaptées d'œuvres littéraires (en 2006, on en dénombrait 47). » Ces BD ont même leur collection particulière chez des éditeurs prestigieux: la collection «Fétiche» chez Gallimard, par exemple, ou « Ex-libris » chez Delcourt, celle-là même où publie Stéphane Heuet. Pour bien comprendre de quoi il s'agit, la BD littéraire doit d'abord être distinguée de deux autres formes connexes avec lesquelles elle coexiste. La première, la moins littéraire des deux, serait celle à la manière du Dracula d'Alberto Breccia, soit une adaptation purement graphique d'un texte littéraire connu, qui fait appel aux cases de la BD sans toutefois y adjoindre la moindre trace de texte, c'est-à-dire ni phylactère ni encadré. Le lecteur est ainsi plongé dans un univers de couleurs et de dessins à partir duquel se construit une trame narrative souvent très fine et même parfois secondaire, posant ainsi que l'illustration, la qualité intrinsèque du dessin, prime sur le récit littéraire, et que l'expérience esthétique recherchée est avant tout une expérience visuelle. L'éditeur Rackham, qui publie en 2006 une réédition du 
Dracula de Breccia, a en toute logique joint à l'album proprement dit le croquis du dessinateur, montrant par là à quel point l'esthétique picturale est primordiale dans ce genre d'adaptation de la littérature. La deuxième pratique, beaucoup plus courante et beaucoup plus ancienne, est le roman illustré, c'est-à-dire le compagnonnage entre l'intégralité d'un texte littéraire et différents dessins d'un artiste à la notoriété variable. Voyage au bout de la nuit de Tardi ou, plus récemment, l'illustration de L'Étranger d'Albert Camus publiée dans la collection "Futuropolis » de Gallimard, en sont des exemples typiques. Dans ce dernier livre, qui demeure un cas d'espèce, la coexistence entre le narratif et l'illustration est produite d'une manière telle que le dessin est au service du récit, qui se trouve d'ailleurs dans son intégralité, comme le souligne la page couverture du livre. Aucune ambiguïté n'est ainsi possible : les dessins de José Muñoz sont au service du récit camusien, et non l'inverse. Les illustrations de Muñoz consistent essentiellement à représenter des moments de la narration de sorte que le lecteur a sous les yeux des images possibles, des visages et des gestes dont la référence exacte et le modèle premier appartiennent, toutefois, à la trame narrative du roman, qui est le réel dépositaire du sens. C'est le texte qui informe le dessin monochrome de Muñoz, qui se caractérise par cette forme de statisme incapable de saisir l'entièreté du récit. Telle qu'elle s'observe aujourd'hui, cette pratique s'inscrit de moins en moins dans une logique pécuniaire mais, au contraire, dans celle d'une démarche esthétique pour spécialistes amoureux des beaux-arts et, surtout, friands de distinction. La forme du livre, sans parler de son prix souvent exorbitant, et de son tirage limité, détonne face à ce que constituait, dans la seconde moitié du XIXe siècle, cette pratique tapageuse à finalité 
commerciale. Le livre illustré semble s'être définitivement métamorphosé en un livre d'art au tournant du XXIe siècle, à l'exemple du Don Quichotte illustré en 1998 par le peintre français Gérard Garouste, objet d'art fabriqué pour les collectionneurs et certainement l'un des ouvrages les plus achevés dans ce genre.

\section{Définir la BD littéraire}

La bande dessinée littéraire répond, quant à elle, à d'autres particularités. Dans son article «Littérature et bande dessinée. Enjeux et limites », Jan Baetens énonce un certain nombre de critères pour la définir. Il s'agit d'abord d'une œuvre hybride qui a recours aux usages courants de la $\mathrm{BD}$, à l'intérieur desquels s'adapte et se fusionne une œuvre littéraire connue. Cette bande dessinée ne se limitera pas à "plaquer" des images sur des textes ou [...] remplir les cases prédécoupées d'une trame narrative déjà entièrement bouclée par un scénario préexistant, conçu et élaboré hors de tout dialogue avec le dessinateur » (p. 4). «Un "bon" récit visuel, précise-t-il, dans le champ de la bande dessinée, est un récit né de la friction créatrice entre deux médias » (p. 4). Cette friction sera positive si elle permet au créateur de garder un équilibre entre l'illustration et la narration, sans que l'une des dimensions n'ait préséance sur l'autre: autrement dit, sans complètement s'affranchir du récit, la BD idéale parviendrait à «rejeter la tutelle du texte» (p.4), de sorte que la nouvelle dialectique mise en place par cette confrontation entre texte et image finirait par produire une œuvre distincte, si ce n'est originale. Grâce aux ressorts formels propres à la $\mathrm{BD}$, une nouvelle lecture de l'œuvre serait dès lors possible. L'exemple de la BD 
L'Étranger, cette fois de Jacques Ferrandez, publiée dans la collection «Fétiche » de Gallimard, et qui peut être considérée comme une réussite du genre, se caractérise par le fait qu'elle met l'accent davantage sur le bédéiste que sur le texte de Camus, comme c'était le cas avec José Muñoz. Ferrandez domine désormais la page couverture dans un rapport qui n'implique en rien l'asservissement au texte de L'Étranger. Le récit dynamique ne cherche pas à couvrir l'intégralité du texte et laisse une place importante aux dessins, auxquels se surajoute du texte qui ne surcharge que très rarement les cases. Ce qui est représenté résulte ainsi d'une interprétation du texte qui laisse une part non négligeable à la subjectivité du bédéiste. L'image est mobile et elle favorise la focalisation sur la séquence et non sur le texte lui-même, de sorte que la BD acquiert sa propre unité et, par là, son autonomie face au roman adapté. Grâce au rythme des cases, le dessin devient dynamique et finit par devenir le principal pourvoyeur du sens. La question reste à savoir si le récit proustien permet un tel transfert et une telle dynamique et, parallèlement, si Stéphane Heuet est parvenu à trouver un réel équilibre dans son adaptation. Camus, il va sans dire, n'est pas Proust: l'unité d'action, le nombre restreint de personnages, la large place qu'occupent les décors et les paysages, sans nommer l'écriture blanche camusienne, semblent autant d'éléments qui facilitent l'adaptation par la BD. Les interminables introspections du narrateur de La Recherche, qui s'ajoutent à l'éclatement temporel de l'action, aux sinuosités narratives et à une multitude de personnages qui évoluent dans une chronologie des plus bigarrées, s'apparentent à des défis d'une tout autre nature, tant ces éléments semblent a priori incompatibles avec la forme traditionnelle qui caractérise la bande dessinée. 


\section{Proust en BD}

La première remarque sur les bandes dessinées de Stéphane Heuet est que son adaptation évolue au cours des cinq albums publiés jusqu'à maintenant. Au sujet de Du côté de chez Swann, dont l'édition intégrale a été publiée récemment, plusieurs remarques s'imposent, tant il semble que l'auteur n'a pas encore trouvé l'équilibre dans son adaptation du roman. Sur la page de couverture d'abord, le nom de Proust prédomine par sa taille sur le nom minuscule du bédéiste, qui est ici écrasé par le gigantisme et le prestige de l'œuvre. Cette particularité ne changera pas plus tard avec les deux albums que compose $\grave{A}$ l'ombre des jeunes filles en fleurs, comme si Heuet tenait, malgré l'évolution de son travail, à souligner, indépendamment de son mode d'adaptation, une hiérarchie des différentes pratiques artistiques, au sommet de laquelle Proust trône évidemment. Cette domination, cependant, est encore plus marquée dans les trois premiers albums. L'édition intégrale de $D u$ côté de chez Swann se manifeste aussi par le fait que cet album contient, caractéristique étrange pour une BD, un appareil critique, des notes en fin de volume, un arbre généalogique de la famille réelle de Marcel Proust, la représentation iconographique de chaque personnage et, surtout, une carte représentant Paris à la fin du XIXe siècle qui, en plus de sa vertu toute didactique, insiste sur le caractère référentiel de l'œuvre romanesque. Adapter Proust devient, du moins dans cet album, l'équivalent de faire de LaRecherche un texte dont les descriptions renverraient à un Paris historique et réel, que les dessins essaieraient tant bien que mal de représenter, Heuet ayant d'ailleurs expliqué qu'il faisait d'abord un travail d'«enquêteur» (2012, p.148), qu'il se référait à des 
photographies de Félix Tournachon (dit Nadar), à des tableaux des peintres du XIXe siècle et à des plans précis du Baron Haussmann pour dessiner le décor dans lequel évoluent les personnages. En plus de sa visée pédagogique, le travail de Stéphane Heuet vise, souligne-t-il, à permettre «à beaucoup de ceux qui ont déjà lu La Recherche de voir à quoi pouvaient ressembler les lieux et monuments qui ont inspiré Combray, Balbec, Doncières, le Paris de Swann, ainsi que la rue La Pérouse, les œuvres d'art évoquées ou partiellement inventées, etc. » (p. 145-146) Cette volonté d'illustrer non pas tant l'univers poétique de La Recherche que la France dans laquelle vivait Proust, comme si l'un était l'équivalent de l'autre, s'accompagne aussi d'un vif désir de fidélité à l'égard du roman, qui se réalise par une surabondance de textes à l'intérieur des premières BD. En plus des bulles qui sont omniprésentes, Heuet a recours continuellement à des cases de couleur «coquille d'œuf » dans lesquelles s'insère la " voix off » du narrateur, qui est nul autre que le narrateur de La Recherche (Heuet, 2012, p. 147). « Je travaille, explique-t-il, à l'envers par rapport à mes collègues auteurs de BD: textes d'abord, dessins ensuite...» (p. 147), méthode qui, au final, a pour conséquence d'alourdir la $\mathrm{BD}$, de mettre sous tutelle les mécanismes propres à ce type de création de sorte qu'illustrer Proust est indissociable de cette incohérence qui consiste à laisser la plus large place possible à l'intérieur de chacune des cases au texte proustien lui-même. Cette inversion est à quelques reprises poussée aux limites de l'absurde lorsque, dans certaines cases, le dessin de Heuet finit par disparaître pour laisser toute la place à la narration de Proust, au texte tel qu'il existe dans le roman, faisant de la narration le seul moteur capable de propager le sens, ce qui 
annule le principe premier, inhérent au projet de Heuet, de changer de support formel et de mettre La Recherche en BD.

C'est peut-être cette incohérence qui rend la version intégrale de $D u$ côté de chez Swann tellement incongrue : cet album ne semble être ni un roman illustré, ni une BD littéraire, ni une suite de dessins inspirée des divagations de Swann. Sans donner à lire l'intégralité du texte proustien, les cases sont tellement surchargées que la lecture, si elle n'est pas difficile, demeure toujours laborieuse et même rebutante. J'avance l'hypothèse suivante pour expliquer cette maladresse initiale : c'est autant le prestige de Proust, la nature de l'intrigue amoureuse et les réminiscences continuelles qui scandent ce premier tome de La Recherche qui auraient écrasé le travail créatif du bédéiste. Heuet semble pétrifié devant La Recherche, à l'exemple de l'adaptation cinématographique de Madame Bovary faite jadis par Claude Chabrol, dans laquelle, au lieu de faire du cinéma, Chabrol avait eu recours à une "voix off » qui, à tout instant, lisait de longs passages du livre, comme si ces dits passages étaient à la fois nécessaires à la compréhension de l'œuvre et intransposables dans une autre forme que celle de l'écriture. Dans les trois premiers albums, Heuet est prisonnier de La Recherche. Le texte proustien est encore son maître, ce qui explique pourquoi il ne fait pas encore confiance à son potentiel de dessinateur. Sa libération se réalise seulement au moment où la vie de Swann n'est plus le centre du récit, ce qui coïncide avec le moment où il finit par modeler sa démarche artistique sur les principes de base de la BD et sur sa forme des plus traditionnelles, soit une bande dessinée en 48 pages (48CC), qui cherche par tous les moyens à faire l'économie du texte et par laisser enfin le haut du pavé au dynamisme de la BD (Baetens, 2012, p. 179 et passim). 
Ainsi, à partir de Noms de pays: le nom, une lente métamorphose se produit dans la BD proustienne. Le lecteur assiste à un double mouvement d'élagage narratif et d'autonomisation du dessin qui, conjointement, transforme les aventures du narrateur, de sorte que l'expérience intérieure s'efface peu à peu pour laisser place aux aléas de la vie d'un jeune homme. Il ne s'agit plus de représenter les névroses d'un homme d'âge mûr pathologiquement attaché à une lorette qui ne lui plaisait pas et qui n'était pas « de son genre », mais les vaet-vient du narrateur au moment où, jadis, il s'éveillait aux attraits de son entourage féminin, manifestement beaucoup plus dégourdi que lui. Même si, il va de soi, les pérégrinations d'un jeune garçon s'adaptent, sans doute, beaucoup plus facilement en BD que les digressions intérieures d'un névropathe jaloux épris d'une cocotte sur le retour, ce renouvellement de la $\mathrm{BD}$ est indissociable d'une modification formelle importante. Il s'agit du dépouillement des cases, qui se fait alors au détriment de la voix narrative qui, elle, ne disparaît pas complètement des albums, mais s'amenuise pour n'énoncer que le nécessaire d'une trame de plus en plus effacée et désormais suggérée par les dessins, et non plus par la retranscription servile de la phrase proustienne. Au lieu de laisser voir le texte, Heuet laisse désormais parler ses dessins de manière que l'entreprise qu'il se donne, soit de mettre Proust en BD, devienne soudainement pertinente. Mise en bande dessinée, cette partie de La Recherche s'apparente dès lors à un roman d'éducation dans lequel un jeune homme n'est plus confronté à la contingence d'une mémoire tyrannique mais à l'indifférence de jeunes filles, aux aléas des vacances d'été, à une quête identitaire, aux hasards de l'amour, sans parler, il va sans dire, de l'incompréhension de ses parents et de sa curiosité 
sexuelle grandissante. J'insiste aussi pour dire que cette partie de La Recherche, qui laisse une large place à l'enfance, semble mieux s'adapter aux contraintes de la BD, comme si le genre exigeait ce type d'atmosphère juvénile pour réaliser pleinement son potentiel. Dès lors, l'univers pictural qui se dégage des derniers albums de Heuet se caractérise par la naïveté, la fraîcheur, l'innocence et la spontanéité, qui demeurent l'opposé de l'atmosphère souvent lugubre des adaptations cinématographiques que l'on connaît : pensons à celle de Volker Schlöndorff avec Jemery Irons dans le rôle de Swann, adaptation, évidemment, qui ne laisse aucune place à l'enfance, à la naïveté du regard et à l'éveil sexuel d'un jeune homme naïf prisonnier d'un milieu décadent. À l'instar de Moby Dick de Melville qui était considéré, à l'origine, comme un ouvrage spécialisé sur la vie des cétacés ou une fable théologique sur la persistance du Mal, mais qui est devenu, en raison de ses multiples adaptations en films, mais surtout en BD et en dessins animés, un roman de jeunesse, " on ne peut nier, avance Jan Baetens, que son adaptation [celle de Stéphane Heuet] a changé, durablement peut-être, notre perception de Proust (lequel doit être maintenant en train de devenir pour certains un auteur pour enfants) » (2004, p. 263). Il reste à voir ce que donnera la suite. Sodome et Gomorrhe, s'il est adapté tel que prévu, ne s'adressera sans doute pas aux tout-petits, mais force est de constater qu'une fois allégé, dépouillé, départi de la grandiloquence des phrases proustiennes, débarrassé de ses considérations philosophiques sur la vacuité du monde et sur l'irrémédiable travail du temps, autrement dit une fois ramené à sa plus fine trame narrative, il pourrait traiter des problèmes liés à l'orientation sexuelle, à l'exemple de $\grave{A}$ l'ombre des jeunes filles en fleurs, transformant ainsi ce récit en une BD pour jeunes 
adolescents qui se questionnent sur les caprices de leur propre libido. C'est en l'obligeant à se départir de la phrase proustienne que la BD permet à Heuet de proposer une lecture radicalement différente de La Recherche. J'ignore, encore une fois, ce que donneront les autres albums si un jour ils sont publiés, mais espérons que Stéphane Heuet laissera une large place aux dessins et qu'il minimisera l'apport de la voix off et des phylactères de manière à donner à voir un autre Proust, celui auquel ne s'attendait pas jusqu'à maintenant le lecteur usuel de La Recherche, un Proust dégarni, dépouillé, quoiqu'aussi, peutêtre, un peu squelettique.

\section{Entre trahison et relecture}

Dans Proust est une fiction, François Bon avance au sujet de La Recherche un principe de lecture qui repose sur l'axiome fondamental du purisme en littérature :

Ainsi va La Recherche, phrase par phrase, strate narrative après strate narrative dans une horlogerie qui d'abord éclate puis se stabilise, enfin exhibe au plus net chacun de ses rouages, pour décrocher ses pages d'anthologie, qu'il n'est plus possible de détacher de l'ensemble sans tout perdre. (2013, p. 174)

Métamorphoser Proust en lecture pour enfants, ou même pour adolescents un peu dégourdis, conduit à la suppression à la fois de la complexité de la narration proustienne et du caractère instable, fluide, bigarré, des passions et des émotions qui définissent les multiples personnages et dont rend compte cette dite narration. Robert, marquis de Saint-Loup-en-Bray, n'est pas Saint-Loup sans sa cocaïnomanie obsessionnelle, sa bisexualité et sa passion pour des perversités de moins en moins naturelles. Les jeunes filles en fleurs seraient très 
éloignées de la sensibilité des jeunes filles proustiennes sans le polymorphisme du surprenant désir de leur épiderme. Que crée le véritable charme d'Odette, si ce n'est la persistance, en elle, des penchants irréformables de Miss Sacripant? Et que dire des goûts d'Albertine, de ceux de la femme de chambre de la Baronne de Putbus et du désir de plus en plus marqué du narrateur à l'égard des très jeunes filles à mesure qu'il vieillit et que le récit s'achemine vers la fin? Si la mise en BD de La Recherche accroît l'accessibilité de l'œuvre à un jeune public considéré ex cathedra comme inculte, espérance que rien d'ailleurs ne garantit, il demeure que cette adaptation fait du même coup disparaître plusieurs singularités du récit de Proust. L'univers de La Recherche n'a pas le même intérêt sans l'apport de ces actions qui se tissent dans l'arrière-scène de la vie mondaine, ce qui transforme souvent le narrateur, et en même temps le lecteur, en une espèce de voyeur avide de comprendre les cryptiques manifestations des personnalités véritables : mademoiselle Vinteuil qui jouit en crachant sur la photo de son père; l'enfance d'Odette prostituée à de riches voyageurs anglais; Jupien, polymorphe, en admiration devant le gros pétard de Charlus; et ce dernier, adepte de la perversion à l'état pur, de la flagellation et de l'aspect mâle, bûcheron, combien viril et énergique, des Canadiens français! Ce que tend à supprimer la $\mathrm{BD}$ a des répercussions sur la manière dont se présentent ces caractéristiques, car pour se réaliser adéquatement dans la forme qui est la sienne, elle doit se dégager $\mathrm{du}$ vaste répertoire métaphorique qui rend compte justement de cette peinture des profondeurs afin que le lecteur comprenne les diverses orientations des personnages et les conséquences de ces orientations sur leur vie, leurs goûts et leurs intérêts respectifs. Les interactions sociales deviennent 
inséparables de l'influence de ces dites licences sur les amours, les amitiés, les rencontres, les déceptions, les mésententes, les joies et les tristesses, de sorte que tout l'arrière-fond de l'univers proustien est à la fois très immoral et hautement métaphorique, deux caractéristiques d'emblée incompatibles avec la visée de cette BD et ses caractéristiques formelles, quoique toutes les deux paradoxalement essentielles pour déchiffrer la motivation de tous ceux qui évoluent dans La Recherche, pour comprendre leurs penchants inavouables, laissant ainsi à découvert, pour celui qui sait bien lire, le territoire interdit de l'intimité, des fantasmes et de l'irrationalité de la chair.

Il reste toutefois que cette trahison inévitable ne condamne pas pour autant cette relecture et ce projet, décidément audacieux, pour ne pas dire un peu fou, de métamorphoser Proust en BD pour jeunes. En raison du travail qu'il accomplit, Stéphane Heuet met en lumière une dimension de l'œuvre proustienne qui n'est traditionnellement pas la principale qui surgit au moment de la fréquentation de La Recherche. Par la $\mathrm{BD}$, un déplacement s'opère vers tout ce qui concerne l'enfance du narrateur, son apprentissage, son inconscience et, surtout, sa curiosité naïve. Le personnage principal se heurte alors à la complexité d'une société dont la nature première lui échappe instinctivement et dont rien, dans son éducation, ne le prépare à saisir la retorse mécanique. Autrement dit, pendant un moment marquant dans sa propre évolution, rien ne correspond à la magie de ses rêves, écart que la transposition du récit en $\mathrm{BD}$ parvient à souligner magistralement en faisant ressortir l'importance de ces années d'apprentissage sur les multiples dénouements de La Recherche. Dans les deux tomes qui constituent À l'ombre des 
jeunes filles en fleurs, le narrateur se métamorphose en une espèce de Tintin, dont la physionomie le situe très loin du jeune décadent repu ou de l'écrivain malade étendu sur un lit. Il court, il bouge, il se questionne, il est le sujet d'une interminable quête dont il ne saisit, à vrai dire, que très peu la nature, car l'essentiel lui échappe. Indépendamment de ses tracas, les cases, les phylactères et l'esthétique juvénile de la BD finissent néanmoins par donner à ce jeune homme une gaité insoupçonnée, une joie de vivre admirable que la phrase proustienne finit peut-être trop souvent par occulter tant elle est dense et symptomatique d'une maturité artistique. Le puriste verra sans doute dans cette refocalisation qu'implique presque naturellement la bédéisation du texte une trahison impardonnable; le lecteur qui se laissera prendre à l'exercice et à la lecture des cinq tomes de Stéphane Heuet finira, par contre, à voir l'ensemble de La Recherche sous un nouvel éclairage et à ajouter, au lot des interprétations courantes, une dimension supplémentaire: un texte d'une grande naïveté, teinté de nostalgie et de romantisme, exaltant un court moment de la vie où un jeune homme s'en va à l'aventure, sans arrière-pensées, ignorant, encore pour un instant, tout de la complexité des désirs. 


\section{Bibliographie}

BAETENS, Jan. (2004), "La bande dessinée "littéraire": une nouvelle chance pour la littérature, un danger pour la bande dessinée », Contemporary French Civilization, vol. 28, n², p. $253-272$.

—. (2009), "Littérature et bande dessinée. Enjeux et limites », Cahiers de narratologie, $\mathrm{n} \times 16, \quad$ p. 1-12, <http://narratologie.revues.org/974?lang=en>.

-. (2012), «Marcel Proust en 48CC», Marcel Proust aujourd'hui, no 9, p. 173-183.

Bon, François. (2013), Proust est une fiction, Paris, Seuil.

Flaubert, Gustave. (1991), Correspondance, t. 3, Paris, Gallimard, coll. «Bibliothèque de la Pléiade».

HEUET, Stéphane. (2012), « La Recherche en BD », Marcel Proust aujourd'hui, no 9, p. 145-172.

LABÉ, Yves-Marie. (2008), "Les bulles et les lettres », Le Monde des livres, 27 mars, <http://www.lemonde.fr/livres/article/2008/03/27/lesbulles-et-les-lettres_1027863_3260.html>.

Proust, Marcel. (2000), À la recherche du temps perdu. À l'ombre des jeunes filles en fleurs, vol. 1, adaptation de Stanislas BRÉzET et Stéphane HEuET, dessin et couleur de Stéphane HEUET, Paris, Delcourt.

-. (2002), À la recherche du temps perdu. À l'ombre des jeunes filles en fleurs, vol. 2, adaptation de Stéphane HEUET et 
Stanislas BRÉzET, dessin et couleur de Stéphane HEUET, Paris, Delcourt.

- . (2013), À la recherche du temps perdu. Combray - Un amour de Swann - Noms de pays: le nom, adaptation et dessin de Stéphane HEUET, Paris, Delcourt.

\title{
Résumé
}

Cet article situe l'adaptation d'À la recherche du temps perdu en bande dessinée faite par Stéphane Heuet par rapport à la tradition du roman illustré et de la bande dessinée littéraire, pour ensuite s'attarder sur les caractéristiques de cette transposition qui a elle-même connu des modifications en cours de route. Heuet a en effet renouvelé son art de bédéiste au fil des tomes pour des raisons liées à la nature du texte proustien et aux contraintes de la bande dessinée, dont la forme en vient à imposer une autre lecture de La Recherche, de ses personnages et de leurs actions.

\begin{abstract}
This article studies Stéphane Heuet's graphic novel adaptation of Proust's In Search of Lost Time through the tradition of literary comics and illustrated novels. It then dwells on the characteristics of this transposition, which has experienced many modifications along the way. Heuet has indeed renewed his art as a cartoonist over the different tomes of his adaptations for reasons related to the nature of the proustian text as well as the constraints of the graphic novel, whose form imposes a new reading of La Recherche, its characters and their actions.
\end{abstract}

